\title{
C-Jun N-Terminal Kinase Signaling Inhibitors Under Development
}

\author{
Sun-Young Han \\ Drug Discovery Division, Korea Research Institute of Chemical Technology, Daejeon 305-343, Korea
}

Received February 6, 2008; Revised March 26, 2008; Accepted March 27, 2008

\begin{abstract}
Targeting protein kinases has been active area in drug discovery. The c-Jun $\mathrm{N}$-terminal kinases (JNKs) have also been target for development of novel therapy in various diseases, since the roles of JNK signaling in pathological conditions were revealed in studies using jnk-deficient mice. Small molecule inhibitors and peptide inhibitors are identified for therapeutic intervention of JNK signaling pathway. SP-600125, an anthrapyrazole small molecule inhibitor for JNK with high potency and selectivity has been widely used for dissecting JNK signaling pathway. CC-401 is the first JNK inhibitor that went into clinical trial for inflammation and leukemia. Inhibitor for mixed lineage kinase (MLK), CEP-1347 also negatively regulates JNK signaling, and tried for potential use in Parkinson's disease. Cell-permeable peptide inhibitor D-JNKI-1 is being developed for the treatment of hearing loss. The current status of these JNK inhibitors and safety issue is discussed in the minireview.
\end{abstract}

Key words: JNK, Inhibitors, SP-600125, CC-401, AS-602801, CEP-1347, D-JNKI-1.

\section{INTRODUCTION}

One of the most active areas in drug development is targeting protein kinases. Out of 518 protein kinases identified from human genome project (Manning et al., 2002), quite a few kinases are known to be involved in signal transduction pathway. And dysregulation of the signaling pathway due to the overexpression, mutation, or deletion of protein kinases are related to pathophysiological process. In practical point of view for drug development, kinases are easy to inhibit using small molecules. The ATP binding pocket shared by all protein kinases is binding site for small molecules and serves as a pharmacophore. As results of efforts for

Correspondence to: Sun-Young Han, Drug Discovery Division, Korea Research Institute of Chemical Technology, 19, Sinseongno, Yuseong-gu, Daejeon 305-343, Korea

E-mail: syhan@krict.re.kr

Abbreviations: JNK, c-Jun N-terminal kinase; ATP, adenosine 5'-triphosphate; MLK, mixed lineage kinase; EGFR, epidermal growth factor receptor; VEGFR, vascular endothelial cell growth factor receptor; MAPK, mitogen-activated protein kinase; MAP2K/MKK, MAPK kinase; MAP3K, MAPK kinase kinase; MEK, MAPK-ERK kinase; ERK, extracellular signalregulated kinase; $\mathrm{Ki}$, inhibitory constant; IKK, IKB kinase; $\mathrm{IC}_{50}$, concentration of compound to achieve $50 \%$ inhibition; CDK, cyclin-dependent kinase; MPTP, 1-methyl-4-phenyl tetrahydropyridine; JBD, JNK-binding domain; JIP, JNK-interacting protein; IL-1 $\beta$, interleukin-1 $\beta$. drug development targeting kinases, several drugs are on the market. Since the first kinase inhibitor on the market imatinib (Gleevec) for treatment of leukemia, several kinase inhibitors are launched recently. These include drugs targeting epidermal growth factor receptor (EGFR), vascular endothelial cell growth factor receptor (VEGFR), and second generation bcr-abl inhibitors. Also small molecules targeting other kinases are under active development by pharmaceutical companies. JNK signaling pathway is one of the molecular targets for therapeutic purpose.

JNK signaling pathway has been a focus of attention for therapeutic target for its diverse roles in cellular processes (for review, refer to Davis, 2000; Weston and Davis, 2002). JNK was initially purified as a protein activated by a variety of stress signals (Kyriakis and Avruch, 1990). Other group independently identified JNK for its affinity to C-Jun N-terminal domain (Hibi et al., 1993). Later this kinase was molecularly cloned and found to be a member of the mitogen-activated protein kinase (MAPK) group. Like other MAPK subfamily, JNK signaling is transmitted by way of three-kinase signaling module comprised of MAPK, MAP2K, and MAP3K (Fig. 1). Members of the JNK family are encoded by three genes (jnk1, jnk2, and jnk3), which are alternatively spliced to yield ten isoforms (Barr and Bogoyevitch, 2001; Bennett et al., 2001).

Also the role of JNK pathway in pathological condi- 


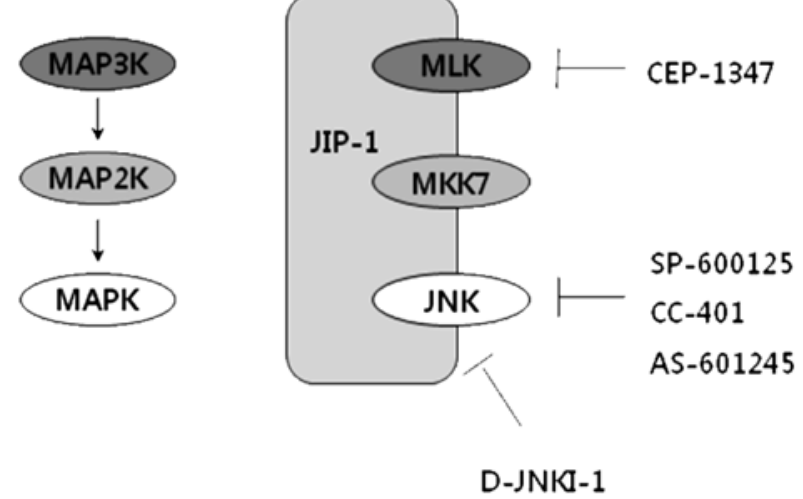

Fig. 1. JNK signaling cascade and inhibitors. JNK signaling pathway is activated by three-kinase signaling module composed of MAPK, MAP2K, and MAP3K, like other MAPK family. For JNK family, the kinase module is composed of JNK1, JNK2, and JNK3 for MAPK, MKK4 and MKK7 for MAP2K, and diverse MAP3Ks including MLKs. The components of the signaling modules are organized by binding to scaffold protein. JIP-1 is a scaffold protein organizing MLK, MKK7, and JNK. JNK signaling inhibitors are indicated for their site of action. CEP-1347 is an ATP-competitive inhibitor for MLK activity. SP-600125, CC-401, and AS-601245 are small molecule inhibitors for JNK activity. D-JNKI-1 is a cell-permeable peptide inhibitor containing the 20 peptide sequence of JIP-1. Figure is adapted from Borsello and ForIoni (2007).

tion has been demonstrated in animal models including jnk-deficient mice. Studies of JNK signaling pathway suggested JNK can be a therapeutic target for diseases including inflammation, neurodegenerative diseases, cancer, obesity, and diabetes. Manning and Davis wrote an excellent review for this topic (Manning and Davis, 2003). Here in this review, current development status of JNK signaling inhibitors will be described along with safety issues of drugs targeting JNK.

\section{INHIBITORS OF JNK SIGNALING CASCADE}

JNK signaling pathway is known to be very important in various pathological conditions. However, there is very short list for small molecule inhibitors under active development targeting JNK protein directly compared to other kinases (Table 1). And most of the JNK inhibitors reported in patent literatures are byproducts from search of p38 MAPK inhibitors (Harper and LoGrasso, 2001; Manning and Davis, 2003). These may reflect the difficulty of finding selective inhibitors for JNK. Here I will describe the first JNK selective inhibitor SP-600125 and other direct JNK inhibitors under active development. Additionally, MLK inhibitor CEP-1347 and peptide inhibitor D-JNKI-1 will be discussed (Fig. 1). The JNK signaling inhibitors are listed in Table 1 together with development status and the name of the company developing the agents.

SP-600125. SP-600125 is a selective JNK inhibitor with chemical structure of anthra[1,9-cd]pyrazol-6-(2H)one (Fig. 2). This anthrapyrozole compound was identified using high-throughput biochemical screen by Celgene corporation (previously known as Signal Pharmaceuticals) (Bennett et al., 2001). SP-600125 is reversible ATP-competitive inhibitors, with $\mathrm{IC}_{50}$ of $40 \mathrm{nM}$ for JNK1, $40 \mathrm{nM}$ for JNK2, and $90 \mathrm{nM}$ for JNK3. SP600125 shows selectivity more than 20-fold over 17 other kinases tested. SP-600125 has 300-fold selectivity for JNK compared with extracellular signal-regulated kinases (ERKs) and p38 MAPKs in the MAPK family. However, SP-600125 inhibits the upstream of JNK and p38 MAPK signaling pathway, which are MKK4 (MAPK kinase 4), MKK7, MKK3, and MKK6, with $I C_{50}$ values of between 0.4 and $5 \mu \mathrm{M}$. Thus, the interpretation of the experimental results with high concentration of SP600125 should be done cautiously. Furthermore, report by Bain et al. (2003) indicated that SP-600125 may not be a selective inhibitor for JNK protein, contradicting previous reports. $I_{50}$ values for JNK1 $1 \alpha 1$ and JNK2 22 were weak at $0.1 \mathrm{mM}$ ATP $(5.8 \mu \mathrm{M}$ and $6.1 \mu \mathrm{M}$, respectively). And some other kinases were inhibited to a greater extent than JNK.

When SP-600125 was first reported in 2001 as a JNK inhibitor (Bennett et al., 2001), this compound became instantly popular in studies dissecting the roles of JNK signaling pathway. Unlike ERK or p38 MAPK

Table 1. JNK signaling inhibitors

\begin{tabular}{|c|c|c|c|c|}
\hline Agent & Company & Target & Status & Therapeutic group \\
\hline SP-600125 & Celgene & JNK & & \\
\hline CC-401/JNK-401 & Celgene & JNK & Phase ॥ & Myeloid Leukemia, Inflammatory disease \\
\hline AS-601245 & Merck Serono & JNK & Preclinical & Cystic fibrosis \\
\hline CEP-1347/KT-7515 & Cephalon & MLK & & Parkinson's disease \\
\hline D-JNKI-1/AM-111/XG-102 & Auris Medical & JNK & Phase $|/ I|$ & $\begin{array}{l}\text { Hearing loss } \\
\text { Myocardial infarction } \\
\text { Ischemic stroke }\end{array}$ \\
\hline
\end{tabular}


<smiles></smiles>

AS-601245<smiles>N#C/C(=C1/Nc2ccccc2S1)c1ccnc(NCCc2cccnc2)n1</smiles>

CEP-1347

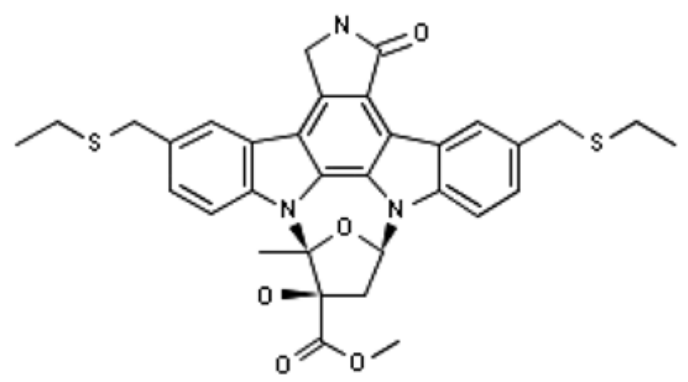

D-JNKI-1

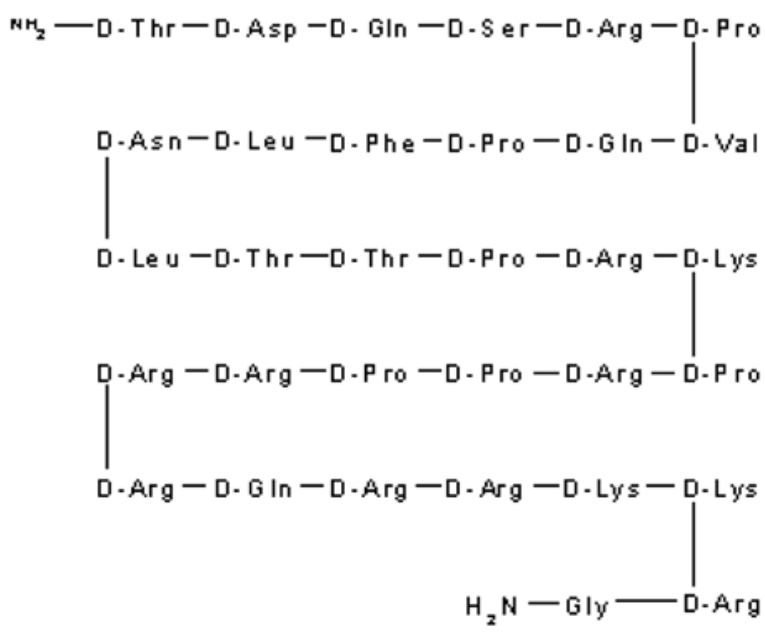

Fig. 2. Chemical structures of JNK pathway inhibitors.

pathway, there were no small molecule inhibitors for JNK pathway. To dissect ERK signaling pathway, MEK1/ MEK2 inhibitor PD98059 and U0126 had been used (Favata et al., 1998). And SB202380 was applied for the study of p38 MAPK signaling pathway at that time (Lee et al., 2000; Salituro et al., 1999). To investigate the JNK pathway, no small molecules were available at that time. Only approach available was the use of domi- nant negative form of JNK, which required transfection process. With the identification of SP-600125 at 2001, this compound was used extensively in combination of dominant negative protein approach in studying cellular biology of JNK pathway.

The potential of SP-600125 in disease application was first shown by the study of Han et al. (2001). To induce arthritis in animal model, Lewis rats were immunized with adjuvant. After 8 days, SP-600125 (30 mg/ $\mathrm{kg} /$ day) was injected subcutaneously for 6 days. SP600125 affected very modestly on the inflammation as shown by foot pad swelling. However, it provided marked protection from joint destruction as indicated by radiographs of rat joint bones. This study suggested that JNK-inhibitory compounds could be clinically effective in the treatment of rheumatoid arthritis. This study was followed by other reports of SP-600125 application in various animal disease models such as tumor xenograft (Ennis et al., 2005), ischemia/reperfusion injury (Ishii et al., 2004; Wang et al., 2007), bacterial otitis media in guinea pig (Furukawa et al., 2007), and smoke inhalation injury in rats (Syrkina et al., 2007).

CC-401. CC-401, also known as JNK-401, is highly selective and ATP-competitive inhibitor of JNK. CC-401 is the first JNK inhibitor which entered into clinical trial by Celgene Corporation. This compound is in phase I trial for the treatment acute myeloid leukemia and inflammatory disease. However, details regarding clinical trials and chemical structure are not disclosed yet. CC-401 inhibits all three forms of JNK isoforms with 25 to $50 \mathrm{nM}$ of $\mathrm{Ki}$ (Ma et al., 2007) and has more than 40fold selectivity for JNK over other related kinases (e.g., p38 MAPK, ERK, and I $\mathrm{B}$ kinase 2).

Two studies were reported for the potential use of CC-401 during transplantation procedures. During the organ transplantation, the organs get damaged from cold ischemic storage followed by warm reperfusion. Rapid JNK activation is observed after ischemia/reperfusion procedures. To prevent injury of the organ during transplantation and to address the role of JNK pathway after transplantation, selective JNK inhibitor CC-401 was used. Pretreatment of CC-401 increased survival after transplantation in both orthotopic rat liver transplantation model (Uehara et al., 2004) and rat hepatic ischemia/ reperfusion model (Uehara et al., 2005). Recent study investigated the potential use of $\mathrm{CC}-401$ in progressive kidney disease by blocking renal fibrosis and tubular apoptosis (Ma et al., 2007). Gavage administration of CC-401 blocked JNK activation and suppressed renal fibrosis and tubular epithelial cell apoptosis in rat unilateral ureteral obstruction model. Furthermore, Ma et al. 
(2007) showed that nonredundant role of JNK1 in tubular cell apoptosis. Only mice with JNK1 deletion not JNK2 deletion showed significant reduction in tubular apoptosis. CC-401 was also reported to be effective in rat model of inflammatory disease called glomerulonephritis (Flanc et al., 2007). CC-401 treatment suppressed glomerular and tubulointerstitial damage in immuneinduced renal injury. Taken the studies together, treatment of CC-401 may be useful with therapeutic benefit, thus the safety data from ongoing phase I clinical trial will be expected.

AS-601245. From the screening of compound library for JNK3 inhibitors, (benzothiazol-2-yl) acetonitrile derivatives were identified as a potent and selective JNK1, JNK2, and JNK3 inhibitors (Gaillard et al., 2005). Further optimization of the compound led to the discovery of AS-601245 (Fig. 2). AS-600245 has $I C_{50}$ of 70 to 220 $\mathrm{nM}$ against JNK isoforms and it exhibited 10 - to 20 -fold selectivity over c-Src, c-Raf, and CDK2 (cyclin-dependent kinase 2). AS-601245 can be administered per oral route, and is under active development by Merck Serono.

In vivo effect of AS-601245 has been evaluated in mouse model of rheumatoid arthritis (Gaillard et al., 2005), myocardial ischemia/reperfusion in rats (Ferrandi et al., 2004), neuronal protection after cerebral ischemia in gerbils (Carboni et al., 2004, 2007). Since jnk3-deficient mice exhibited dramatic protection from neuronal apoptosis induced by excitotoxicity (Yang et al., 1997), it is considered good strategy to use JNK inhibitor for the purpose of neuroprotection. At a dose of $80 \mathrm{mg} / \mathrm{kg}$, AS-601245 reduced neurites damage by $67 \%$ and decreased astrocytes activation by $84 \%$ (Carboni et al., 2007). Also as neurobehavioral measure, AS-601245 improved long-term memory.

CEP-1347. CEP-1347, also known as KT-7515 (for structure, see Fig. 2) is a semisynthetic derivative of K$252 a$, a natural product indolocarbazole of the bacterium Nocardiosis species (Kaneko et al., 1997). At first, CEP-1347 was characterized to have neurotrophic properties (Kaneko et al., 1997). And then it was identified to have inhibitory activity against JNK signaling pathway, but JNK was not a direct target of CEP-1347 (Maroney et al., 1998). Later, the same group identified MLK family as a target of CEP-1347 (Maroney et al., 2001). MLK family belongs to MAP3K that regulates JNK activity, and is consisted of MLK1, MLK2, MLK3, dual leucine zipper kinase, and leucine zipper kinase. CEP-1347 is ATP-competitive inhibitor with $\mathrm{IC}_{50}$ values ranging from 23 to $51 \mathrm{nM}$.
CEP-1347 was developed focusing its activity on blocking cell death of motorneuron, therefore potential use in Parkinson's disease. To mimic human Parkinson's disease in animal model, neurotoxin called MPTP (1-methyl-4-phenyl tetrahydropyridine) is widely used to destroy nigrostriatal dopaminergic neurons (Langston, 1996). Administration of MPTP activated JNK pathway and application of CEP-1347 blocked JNK signaling (Saporito et al., 2000). Also in this MPTP-induced animal model, CEP-1347 protected dopaminergic neurons from apoptosis (Saporito et al., 1999). Based on the encouraging results in the animal model, clinical trials of CEP-1347 to delay disability in early Parkinson's disease were conducted by pharmaceutical company called Cephalon. To everybody's disappointment, treatment of Parkinson's disease with CEP-1347 was not effective in terms of delaying disease progression (Parkinson Study Group, 2007). According to the Parkinson study group who conducted clinical trial and comments by Ahlskog (Ahlskog, 2007) and Waldmeier et al. (Waldmeier et al., 2006), this negative study gave us a few lessons. First, we still don't know enough about cell death mechanism in neurodegenerative diseases. Therapeutic intervention of JNK pathway may be compensated by other mechanisms. This compensation may make CEP-1347 ineffective in Parkinson's disease. Second, current animal model (MPTP-induced neurotoxicity) may not reflect human Parkinson's disease properly. Although, MPTP model provided insight into symptomatic treatment of Parkinson's disease, this model may not be able to predict for delaying progression of Parkinson's disease. A small molecule called TCH346 developed for Parkinson's disease with different mechanism showed good activity in MPTP-induced animal model. However, TCH346 failed to show efficacy in human. This can be an another example for the lack of correlation between MPTP model and human Parkinson's disease (Waldmeier et al., 2006).

$D-J N K I-1 / A M-111 / X G-102$. There are efforts identifying JNK inhibitors other than small molecule approach. D-JNKI-1 is a peptide inhibitor of JNK activity under active development. D-JNKI-1 is based on the peptide sequence of its scaffold protein called JIP-1 (JNK interacting protein-1) protein (Bonny et al., 2001). There is JNK-binding domain (JBD) in JIP-1 protein, and transfection of 20 amino acid sequences from JBD inhibited JNK activity, presumably by blocking access to many of its substrates by a competitive mechanism (Barr et al., 2002; Bonny et al., 2001). This 20 amino acid peptide was further modified to make it cell-permeable. Covalent linkage of 10-amino acid sequence of TAT protein 
from HIV (human immunodeficiency virus) with this peptide enabled intracellular delivery (Schwarze et al., 1999). Furthermore, the peptide was synthesized with all-D retro-inverso form, which is composed of $D$ instead of L-amino acids and synthesized in reverse order (Fig. 2). Although this switch from L-enantiomer decreased the efficacy of JNK-inhibitory effect, half-life of the peptide was markedly increased due to the protection from proteolytic cleavage.

D-JNKI-1 has property of potent activity inside the cells compared with in vitro biochemical activity. $\mathrm{IC}_{50}$ of D-JNKI-1 is $1 \mathrm{mM}$ in vitro biochemical JNK activity assay, whereas $1 \mathrm{nM}$ of D-JNKI-1 is sufficient for protection of pancreatic $\beta$-cell apoptosis induced by IL- $1 \beta$ by $50 \%$ (Bonny et al., 2001). Another group also reported inhibitory effect of peptide sequence derived from JIP-1 protein (Barr et al., 2002). Barr et al. showed that the shorter size (11-amino acid) of JBD domain retained the inhibitory activity. Approach of linking TAT sequence was also used successfully for this smaller size peptide by another group (Guan et al., 2006).

D-JNKI-1 was evaluated for neuroprotective effects in ischemic stroke model in rodents (Borsello et al., 2003; Hirt et al., 2004). D-JNKI-1 peptide is expected to block neuronal apoptosis induced by excitotoxicity during cerebral ischemia. This cell-penetrating peptide showed powerful protection (>90\%), and remarkably D-JNKI-1 was administered 6 12 hour after the onset of ischemia. This study indicated that neuronal death can be blocked 6 12 hour after the onset of stroke, establishing proof of principle for JNK pathway as a target for therapeutic intervention. Additionally, there are reports for the application of D-JNKI-1 for myocardial ischemia-reperfusion (Milano et al., 2007) and use of similar JNK inhibitory peptide to diabetes (Kaneto et al., 2004).

For practical point of view, there are limitations of peptide inhibitor in human therapy: cost and toxicity (Borsello and Bonny, 2004). The cost of producing peptide molecule is very expensive compared with small molecule inhibitor and systemic administration of peptide may be toxic when used in high dosage. Borsello and Bonny (2004) suggested restricting the use of peptide inhibitors in small organs, including eyes and ears to circumvent these problems.

For this reason, D-JNKI-1 is being developed for the treatment of hearing loss. Hearing loss come from cell death of auditory hair cells and auditory neurons caused by noise, infection and ototoxic drugs. The strategy was to block hair cell death by D-JNKI-1-mediated JNK inhibition. Wang et al. (2003) demonstrated that application of D-JNKI-1 prevented nearly all hair cell death and permanent hearing loss in guinea pig ototox- icity model. Furthermore, D-JNKI-1 was effective when applied 12 hours after the sound trauma in animal model (Wang et al., 2007). Based on the effect of D-JNKI-1 on hearing loss in animal model, this peptide is in clinical trial for hearing loss by Auris Medical (Suckfuell et al., 2007) and designated as orphan drug. The granting of orphan drug status gives advantage in developing drugs for rare diseases by way of tax reductions and marketing exclusivity for an extended time.

\section{SAFETY OF JNK SIGNALING INHIBITORS}

During the course of drug development, evaluation of toxicological safety is important part of preclinical development. Also in clinical development, assessing safety is the main purpose of Phase I and Phase II clinical trials. When it comes to signaling pathway inhibitors, the toxicity manifested can be two kinds. One is from the unique characteristic of the specific chemical. In this case, we just need to stop the development of the specific chemical and find other agents. The other kind is mechanism-based toxicity, i.e. the one coming from the inhibition of the signaling pathway. For JNK signaling inhibitor, the toxicity may be manifested by the inhibition of cellular process mediated by JNK signaling. If toxicity from JNK pathway inhibition is a major problem, we may need to reconsider JNK pathway as a therapeutic target or find a way to avoid toxicity like local administration. Until now, three agents CC-401, CEP-1347, and D-JNKI-1 went into clinical trials. The safety data from the studies will be discussed in this chapter.

Our knowledge regarding clinical studies of CC-401 is limited due to the lack of publications of the data. Just from the press release, it is known that double-blind, placebo-controlled phase I study of ascending single intravenous doses are completed. The results from MLK inhibitor CEP-1347 in clinical studies by Parkinson Study Group are found in a few publications (Parkinson Study Group, 2007; Parkinson Study Group, 2004). Oral administration of CEP-1347 was reported to be generally safe and well tolerated in both healthy volunteers and Parkinson's disease patients. Cell-permeable peptide D-JNKI-1 was evaluated for its safety in patients with acute acoustic trauma after exposure to firecrackers on New Year's Eve (Suckfuell et al., 2007). Intratympanic administration of D-JNKI-1 was generally safe and well-tolerated. Although a total of 13 adverse events were reported, the majority involving tinnitus, it seemed to be unrelated to the medication.

It seems too early to tell general safety of JNK signaling inhibitors based on the current clinical data. Although 
safety of CEP-1347 is confirmed in several clinical studies, caution should be taken to extrapolate the results to other JNK inhibitor. Since CEP-1347 does not inhibit JNK directly, direct JNK inhibitor may show different symptoms in terms of adverse effects. In case of DJNKI-1, it was administered locally within the ears, thus systemic effect was not assessed in the phase I/II study (Suckfuell et al., 2007).

\section{CONCLUDING REMARKS}

In this review, current status of drug development for JNK signaling inhibitors is discussed. Novel therapeutic strategy using neuroprotective effects of JNK signaling inhibitor was highly expected, since protection from neuronal apoptosis in jnk3-deficient mice were quite dramatic (Yang et al., 1997). Therefore, failure of translating this research into the therapy of Parkinson's disease using CEP-1347 was quite a disappointment (Parkinson Study Group, 2007). However, lack of efficacy for Parkinson's disease using MLK inhibitor CEP-1347 does not necessarily mean that JNK inhibitors are not applicable to other disorders accompanying neuronal cell death. Efforts should be continued for development of novel therapies using JNK inhibitors in diseases involving neuronal apoptosis. The case of D-JNKI-1 is quite encouraging that investigators found a niche in hearing loss for disease involving neuronal apoptosis. The research progress of CC-401 and AS-601245 during the next few years will be very exciting.

\section{REFERENCES}

Ahlskog, J.E. (2007). I can't get no satisfaction: still no neuroprotection for Parkinson disease. Neurology, 69, 14761477.

Bain, J., McLauchlan, H., Elliott, M. and Cohen, P. (2003). The specificities of protein kinase inhibitors: an update. Biochem. J., 371, 199-204.

Barr, R.K. and Bogoyevitch, M.A. (2001). The c-Jun N-terminal protein kinase family of mitogen-activated protein kinases (JNK MAPKs). Int. J. Biochem. Cell Biol., 33, 1047-1063.

Barr, R.K., Kendrick, T.S. and Bogoyevitch, M.A. (2002). Identification of the critical features of a small peptide inhibitor of JNK activity. J. Biol. Chem., 277, 10987-10997.

Bennett, B.L., Sasaki, D.T., Murray, B.W., O'Leary, E.C., Sakata, S.T., Xu, W., Leisten, J.C., Motiwala, A., Pierce, S., Satoh, Y., Bhagwat, S.S., Manning, A.M. and Anderson, D.W. (2001). SP600125, an anthrapyrazolone inhibitor of Jun N-terminal kinase. Proc. Natl. Acad. Sci. USA, 98, 13681-13686.

Bonny, C., Oberson, A., Negri, S., Sauser, C. and Schorderet, D.F. (2001). Cell-permeable peptide inhibitors of JNK: novel blockers of beta-cell death. Diabetes, 50, 77-82.
Borsello, T. and Bonny, C. (2004). Use of cell-permeable peptides to prevent neuronal degeneration. Trends Mol. Med., 10, 239-244

Borsello, T., Clarke, P.G., Hirt, L., Vercelli, A., Repici, M., Schorderet, D.F., Bogousslavsky, J. and Bonny, C. (2003). A peptide inhibitor of c-Jun $\mathrm{N}$-terminal kinase protects against excitotoxicity and cerebral ischemia. Nat. Med., 9, 11801186.

Borsello, T. and Forloni, G. (2007). JNK signalling: a possible target to prevent neurodegeneration. Curr. Pharm. Des., 13, 1875-1886.

Carboni, S., Boschert, U., Gaillard, P., Gotteland, J. P., Gillon, J.Y. and Vitte, P.A. (2007). AS601245, a c-Jun NH(2)-terminal kinase (JNK) inhibitor, reduces axon/dendrite damage and cognitive deficits after global cerebral ischaemia in gerbils. Br. J. Pharmacol., 153, 157-163.

Carboni, S., Hiver, A., Szyndralewiez, C., Gaillard, P., Gotteland, J.P. and Vitte, P.A. (2004). AS601245 (1,3-benzothiazol-2-yl (2-[[2-(3-pyridinyl) ethyl] amino]-4 pyrimidinyl) acetonitrile): a c-Jun $\mathrm{NH} 2$-terminal protein kinase inhibitor with neuroprotective properties. J. Pharmacol. Exp. Ther., 310, 2532.

Davis, R.J. (2000). Signal transduction by the JNK group of MAP kinases. Cell, 103, 239-252.

Ennis, B.W., Fultz, K.E., Smith, K.A., Westwick, J.K., Zhu, D., Boluro-Ajayi, M., Bilter, G.K. and Stein, B. (2005). Inhibition of tumor growth, angiogenesis, and tumor cell proliferation by a small molecule inhibitor of c-Jun $\mathrm{N}$-terminal kinase. J. Pharmacol. Exp. Ther., 313, 325-332.

Favata, M.F., Horiuchi, K.Y., Manos, E.J., Daulerio, A.J., Stradley, D.A., Feeser, W.S., Van Dyk, D.E., Pitts, W.J., Earl, R.A., Hobbs, F., Copeland, R.A., Magolda, R.L., Scherle, P.A. and Trzaskos, J.M. (1998). Identification of a novel inhibitor of mitogen-activated protein kinase kinase. $J$. Biol. Chem., 273, 18623-18632.

Ferrandi, C., Ballerio, R., Gaillard, P., Giachetti, C., Carboni, S., Vitte, P.A., Gotteland, J.P. and Cirillo, R. (2004). Inhibition of C-Jun N-terminal kinase decreases cardiomyocyte apoptosis and infarct size after myocardial ischemia and reperfusion in anaesthetized rats. Br. J. Pharmacol., 142, 953-960.

Flanc, R.S., Ma, F.Y., Tesch, G.H., Han, Y., Atkins, R.C., Bennett, B.L., Friedman, G.C., Fan, J.H. and Nikolic-Paterson, D.J. (2007). A pathogenic role for JNK signaling in experimental anti-GBM glomerulonephritis. Kidney Int., 72, 698-708.

Furukawa, M., Ebmeyer, J., Pak, K., Austin, D.A., Melhus, A., Webster, N.J. and Ryan, A.F. (2007). Jun N-terminal protein kinase enhances middle ear mucosal proliferation during bacterial otitis media. Infect. Immun., 75, 2562-2571.

Gaillard, P., Jeanclaude-Etter, I., Ardissone, V., Arkinstall, S., Cambet, Y., Camps, M., Chabert, C., Church, D., Cirillo, R., Gretener, D., Halazy, S., Nichols, A., Szyndralewiez, C., Vitte, P.A. and Gotteland, J.P. (2005). Design and synthesis of the first generation of novel potent, selective, and in vivo active (benzothiazol-2-yl)acetonitrile inhibitors of the c-Jun N-terminal kinase. J. Med. Chem., 48, 4596607.

Guan, Q.H., Pei, D.S., Zong, Y.Y., Xu, T.L. and Zhang, G.Y. 
(2006). Neuroprotection against ischemic brain injury by a small peptide inhibitor of c-Jun N-terminal kinase (JNK) via nuclear and non-nuclear pathways. Neuroscience, 139, 609-627.

Han, Z., Boyle, D.L., Chang, L., Bennett, B., Karin, M., Yang, L., Manning, A.M. and Firestein, G.S. (2001). c-Jun N-terminal kinase is required for metalloproteinase expression and joint destruction in inflammatory arthritis. J. Clin. Invest., 108, 73-81.

Harper, S.J. and LoGrasso, P. (2001). Signalling for survival and death in neurones: the role of stress-activated kinases, JNK and p38. Cell Signal., 13, 299-310.

Hibi, M., Lin, A., Smeal, T., Minden, A. and Karin, M. (1993). Identification of an oncoprotein- and UV-responsive protein kinase that binds and potentiates the c-Jun activation domain. Genes. Dev., 7, 2135-2148.

Hirt, L., Badaut, J., Thevenet, J., Granziera, C., Regli, L., Maurer, F., Bonny, C. and Bogousslavsky, J. (2004). D-JNKI1, a cell-penetrating c-Jun-N-terminal kinase inhibitor, protects against cell death in severe cerebral ischemia. Stroke, 35 , 1738-1743

Ishii, M., Suzuki, Y., Takeshita, K., Miyao, N., Kudo, H., Hiraoka, R., Nishio, K., Sato, N., Naoki, K., Aoki, T. and Yamaguchi, K. (2004). Inhibition of c-Jun NH2-terminal kinase activity improves ischemia/reperfusion injury in rat lungs. J. Immunol., 172, 2569-2577.

Kaneko, M., Saito, Y., Saito, H., Matsumoto, T., Matsuda, Y., Vaught, J.L., Dionne, C.A., Angeles, T.S., Glicksman, M.A., Neff, N.T., Rotella, D.P., Kauer, J.C., Mallamo, J.P., Hudkins, R.L. and Murakata, C. (1997). Neurotrophic 3,9bis[(alkylthio)methyl]-and-bis(alkoxymethyl)-K-252a derivatives. J. Med. Chem., 40, 1863-1869.

Kaneto, H., Nakatani, Y., Miyatsuka, T., Kawamori, D., Matsuoka, T.A., Matsuhisa, M., Kajimoto, Y., Ichijo, H., Yamasaki, Y. and Hori, M. (2004). Possible novel therapy for diabetes with cell-permeable JNK-inhibitory peptide. Nat. Med., 10, 1128-1132.

Kyriakis, J.M. and Avruch, J. (1990). pp54 microtubule-associated protein 2 kinase. A novel serine/threonine protein kinase regulated by phosphorylation and stimulated by poly-L-lysine. J. Biol. Chem., 265, 17355-17363.

Langston, J.W. (1996). The etiology of Parkinson's disease with emphasis on the MPTP story. Neurology, 47, S153S160.

Lee, J.C., Kumar, S., Griswold, D.E., Underwood, D.C., Votta, B.J. and Adams, J.L. (2000). Inhibition of p38 MAP kinase as a therapeutic strategy. Immunopharmacology, 47, 185201.

Ma, F.Y., Flanc, R.S., Tesch, G.H., Han, Y., Atkins, R.C., Bennett, B.L., Friedman, G.C., Fan, J.H. and Nikolic-Paterson, D.J. (2007). A pathogenic role for c-Jun amino-terminal kinase signaling in renal fibrosis and tubular cell apoptosis. J. Am. Soc. Nephrol., 18, 472-484.

Manning, A.M. and Davis, R.J. (2003). Targeting JNK for therapeutic benefit: from junk to gold? Nat. Rev. Drug. Discov., 2, 554-565.

Manning, G., Whyte, D.B., Martinez, R., Hunter, T. and Sudarsanam, S. (2002). The protein kinase complement of the human genome. Science, 298, 1912-1934.
Maroney, A.C., Finn, J.P., Connors, T.J., Durkin, J.T., Angeles, T., Gessner, G., Xu, Z., Meyer, S.L., Savage, M.J., Greene, L.A., Scott, R.W. and Vaught, J.L. (2001). Cep-1347 (KT7515), a semisynthetic inhibitor of the mixed lineage kinase family. J. Biol. Chem., 276, 25302-25308.

Maroney, A.C., Glicksman, M.A., Basma, A.N., Walton, K.M., Knight, E., Jr., Murphy, C.A., Bartlett, B.A., Finn, J.P., Angeles, T., Matsuda, Y., Neff, N.T. and Dionne, C.A. (1998). Motoneuron apoptosis is blocked by CEP-1347 (KT 7515), a novel inhibitor of the JNK signaling pathway. J. Neurosci., 18, 104-111.

Milano, G., Morel, S., Bonny, C., Samaja, M., von Segesser, L.K., Nicod, P. and Vassalli, G. (2007). A peptide inhibitor of c-Jun $\mathrm{NH} 2$-terminal kinase reduces myocardial ischemiareperfusion injury and infarct size in vivo. Am. J. Physiol. Heart. Circ. Physiol., 292, H1828-H1835.

Parkinson Study Group (2007). Mixed lineage kinase inhibitor CEP-1347 fails to delay disability in early Parkinson disease. Neurology, 69, 1480-1490.

Parkinson Study Group (2004). The safety and tolerability of a mixed lineage kinase inhibitor (CEP-1347) in PD. Neurology, 62, 330-332.

Salituro, F.G., Germann, U.A., Wilson, K.P., Bemis, G.W., Fox, T. and Su, M.S. (1999). Inhibitors of p38 MAP kinase: therapeutic intervention in cytokine-mediated diseases. Curr. Med. Chem., 6, 807-823.

Saporito, M.S., Brown, E.M., Miller, M.S. and Carswell, S. (1999). CEP-1347/KT-7515, an inhibitor of c-jun N-terminal kinase activation, attenuates the 1-methyl-4-phenyl tetrahydropyridine-mediated loss of nigrostriatal dopaminergic neurons In vivo. J. Pharmacol. Exp. Ther., 288, 421-427.

Saporito, M.S., Thomas, B.A. and Scott, R.W. (2000). MPTP activates c-Jun $\mathrm{NH}(2)$-terminal kinase (JNK) and its upstream regulatory kinase MKK4 in nigrostriatal neurons in vivo. J. Neurochem., 75, 1200-1208.

Schwarze, S.R., Ho, A., Vocero-Akbani, A. and Dowdy, S.F. (1999). In vivo protein transduction: delivery of a biologically active protein into the mouse. Science, 285, 15691572.

Suckfuell, M., Canis, M., Strieth, S., Scherer, H. and Haisch, A. (2007). Intratympanic treatment of acute acoustic trauma with a cell-permeable JNK ligand: a prospective randomized phase I/II study. Acta Otolaryngol., 127, 938-942.

Syrkina, O.L., Quinn, D.A., Jung, W., Ouyang, B. and Hales, C.A. (2007). Inhibition of JNK activation prolongs survival after smoke inhalation from fires. Am. J. Physiol. Lung. Cell Mol. Physiol., 292, L984-L991.

Uehara, T., Bennett, B., Sakata, S.T., Satoh, Y., Bilter, G.K., Westwick, J.K. and Brenner, D.A. (2005). JNK mediates hepatic ischemia reperfusion injury. J. Hepatol., 42, 850859.

Uehara, T., Xi Peng, X., Bennett, B., Satoh, Y., Friedman, G., Currin, R., Brenner, D.A. and Lemasters, J. (2004). c-Jun $\mathrm{N}$-terminal kinase mediates hepatic injury after rat liver transplantation. Transplantation, 78, 324-332.

Waldmeier, P., Bozyczko-Coyne, D., Williams, M. and Vaught, J.L. (2006). Recent clinical failures in Parkinson's disease with apoptosis inhibitors underline the need for a paradigm shift in drug discovery for neurodegenerative 
diseases. Biochem. Pharmacol., 72, 1197-1206

Wang, J., Ruel, J., Ladrech, S., Bonny, C., van de Water, T.R. and Puel, J.L. (2007). Inhibition of the c-Jun N-terminal kinase-mediated mitochondrial cell death pathway restores auditory function in sound-exposed animals. Mol. Pharmacol., 71, 654-666.

Wang, J., Van De Water, T.R., Bonny, C., de Ribaupierre, F. Puel, J.L. and Zine, A. (2003). A peptide inhibitor of c-Jun $\mathrm{N}$-terminal kinase protects against both aminoglycoside and acoustic trauma-induced auditory hair cell death and hearing loss. J. Neurosci., 23, 8596-8607.
Wang, Y., Ji, H.X., Xing, S.H., Pei, D.S. and Guan, Q.H. (2007). SP600125, a selective JNK inhibitor, protects ischemic renal injury via suppressing the extrinsic pathways of apoptosis. Life Sci., 80, 2067-2075.

Weston, C.R. and Davis, R.J. (2002). The JNK signal transduction pathway. Curr. Opin. Genet. Dev., 12, 14-21.

Yang, D.D., Kuan, C.Y., Whitmarsh, A.J., Rincon, M., Zheng, T.S., Davis, R.J., Rakic, P. and Flavell, R.A. (1997). Absence of excitotoxicity-induced apoptosis in the hippocampus of mice lacking the Jnk3 gene. Nature, 389, 865-870. 